\title{
DOCE CUESTIONES FUNDAMENTALES SOBRE LA DIRECCIÓN INTEGRADA DE PROYECTO -PROJET MANAGEMENT- APLICADA A LA CONSTRUCCIÓN
}

\author{
(TWELVE BASIC QUESTIONS ON PROJECT MANAGEMENT APPLIED TO CONSTRUCTION )
}

Rafael de Heredia, Dr. Ingeniero Industrial

Catedrático de la E.T.S. Ing. Industriales. Universidad

Politécnica de Madrid. CONSULTOR

Fecha de recepción: 26-IV-95

ESPAÑA

\begin{abstract}
RESUMEN
En los paises más avanzados, sobre todo del entorno anglosajón como EE.UU., Reino Unido, Canadá, etc., se impone, cada vez con más frecuencia, el empleo de las técnicas de DIRECCIÓN INTEGRADA DE PROYECTO o "Project Management" y DIRECCIÓN INTEGRADA DE CONSTRUCCION o "Construction Management", con el fin de mejorar la eficacia en el proceso constructivo completo.
\end{abstract}

En nuestro país, estas técnicas avanzadas son poco conocidas en general y se aplican de forma esporádica por algunos especialistas y en casos muy singulares.

El presente articulo, escrito en forma de diálogo, constituye un intento de explicar, de forma clara y al mismo tiempo sucinta, los aspectos esenciales de la DIP. PM aplicado a la construcción.

\section{SUMMARY}

In the most developed countries, especially in the AngloSaxon world, namely United States, Great Britain, Canada, etc., the Project Management and Construction Management techniques are being ever more used in order to improve the efficiency of the entire construction process.

In our country these advanced techniques are, generally speaking, not well known. They are applied randomly by some specialists and in very particular cases.

This article, written in the form of a dialogue, is an attempt to explain, clearly though briefly, the essential aspects of PM applied to construction.
Se habla muchode Project Management (PM). También de Dirección Integrada de Proyecto (DIP). Los periódicos en sus secciones de selección de personal, publican anuncios de puestos de trabajo para "Project Managers" o Directores de Proyecto; en algunos carteles de obra comienza a verse, al lado de los técnicos tradicionalmente responsables, el nombre de la entidad que realiza estas funciones de DIP. Por otra parte, se escucha y se lee que las Empresas multinacionales que realizan sus inversiones, incluso en el campo inmobiliario, están dispuestas a mejorar la eficacia del proceso constructivo utilizando bien "Project Management”, bien "Construction Management” (DIC).

Con el fin de divulgar qué es y en qué consiste el "Project Management" (en Español, Dirección Integrada de
Proyecto), me ha parecido conveniente escribir este artículo en forma de un diálogo que dé respuesta a las preguntas que pueden aparecer en un principio a aquellas personas no iniciadas en el conocimiento y utilización de la DIP/DIC.

Este diálogo es el que se presenta a continuación.

1. DENTRO DEL SECTOR DE LA CONSTRUCCIÓN, CADA VEZ SE HABLA MÁS DEL "PROJECT MANAGEMENT" Y DE SU APLICACIÓN A LAS GRANDES PROMOCIONES INMOBILIARIAS, ASÍ COMO A LAS CONSTRUCCIONES INDUSTRIALES. PARA EL LECTOR NO ESPECIALIZADO, PODRÍA DECIRNOS, EN PRIMER LUGAR, ¿EXISTE UNA TRADUCCIÓN PRECISA DE L A EXPRESIÓN 
"PROJECT MANAGEMENT" AL ESPAÑOL ? Y TAMBIÉN, ¿CÓMO PODRÍA RESUMIRSE QUÉ ES Y ENQUÉ CONSISTE EL “PM" ENLÍNEAS GENERALES Y CUÁLES SON SUS OBJETIVOS PRIORITARIOS?; POR ÚLTIMO, ¿EN QUÉ SE DIFERENCIA DE LA DIRECCIÓN FACULTATIVA DE UNA OBRA?

No existe ninguna traducción del término "Management", ni al español ni a ninguna otra de las lenguas latinas. "Management" significa, de forma abreviada, la optimización de recursos mediante su adecuada dirección y en cualquier escalón de una organización, así como la integración de éstos con la finalidad de obtener beneficio. Esto significa la obtención de los resultados previstos.

La traducción que he dado a este término Management es la de Dirección Integrada, por considerar que aunque no es una traducción exacta de la palabra, es la expresión que mejor resume y encierrael concepto de integración vertical y horizontal para la dirección de todos los recursos de que se disponen.

Para explicar en qué consiste el "Project Management", que es la segunda parte de esta pregunta, hay que entrar también en el actual concepto de "Proyecto". Tradicionalmente, y en el campo de la construcción, se considera proyecto el conjunto de planos y otros documentos (Pliego de Condiciones, Memoria y Presupuesto), que definen con mayor o menor exactitud lo que ha de ser una obra y cual será su coste de ejecución.

El concepto actual de Proyecto es muy diferente y mucho más amplio: Proyecto es la combinación de recursos humanos y no humanos reunidós en una organización temporal para conseguir un propósito determinado. Por consiguiente, la Dirección Integrada de Proyecto, entendiendo éste en el sentido de la definición actual, es la dirección de todos los recursos (tiempo, dinero, personas y otros que se puedan utilizar) para conseguir ese "propósito determinado" que se explicita mediante los objetivos a lograr. Estos objetivos, de manerageneral son los referentes al alcance del proyecto, que a su vez da lugar a los de coste de la inversión, plazo de ejecución, calidad (o funcionalidad, o rendimientos, o algún otro concepto análogo) a obtener. Estos son los objetivos principales del Proyecto, de los que a su vez pueden derivarse otros secundarios.

Por tanto, la Dirección Integrada de Proyecto se diferencia de laDirección Facultativa tradicional en que ésta tiene por misión sólo y únicamente la obtención de la calidad definida en el proyecto, entendido en su sentido tradicional, mientras que la Dirección Integrada de Proyecto trata de obtener, al menos, los objetivos principales (alcance, coste, plazo y calidad) que se fijan en el comienzo de la vida del Proyecto para hacer que éste sea viable, $y$, a la vez, procurar la satisfacción del Propietario/Promotor y de las partes interesadas en los resultados del Proyecto.
2. ¿EXISTE ALGUNA DIFERENCIA CON LA COORDINACIÓN Y SEGUIMIENTOQUE LLEVAN A CABO ALGUNAS EMPRESAS PRIVADAS O PÚBLICAS PARA SUS PROPIAS PROMOCIONES SOBRE LOS DIFERENTES AGENTES QUE INTERVIENEN EN EL PROCESO CONSTRUCTIVO?; ¿PODRÍA PONER ALGÚN EJEMPLO QUE MUESTRE LAS POSIBLES DIFERENCIAS?.

Supongo que la preguntase refiere a métodos de coordinación y seguimiento entre las distintas partes actoras en la ejecución de un Proyecto. En tal caso, sí puedo decir que existe diferencia entre un enfoque tradicional y un enfoque por Dirección Integrada de Proyecto, ya que habitualmente los técnicos que intervienen en una obra enfocan todo su trabajo hacia la coordinación y control de aspectos técnicos de la ejecución y, en todo caso, y ya no con enfoque muy tradicional, también se ocupan de la coordinación de estas partes actoras para la obtención del plazo, aunque de manera normal, esta actividad se realiza de manera segregada e inconexa con la dirección facultativa; exactamente pasa esto con el control y seguimiento del coste de la inversión, ya que con frecuencia en lugar de su control y seguimiento para corregir las desviaciones, lo que se hace es pura contabilidad o control presupuestario que es lo que significa, por ejemplo, el control y verificación de certificaciones de obra ejecutada.

La diferencia fundamental entre el sistema de Dirección Integrada de Proyecto -DIP- y el tradicional, es que todas las actividades de la DIP constituyen un sistema y por lo tanto están interrelacionadas entre sí y por otra parte, que éstas se llevan a cabo a través de un equipo, cuya figura principal es el Director de Proyecto que tiene como responsabilidad principal la obtención del objetivo global del Proyecto que es, dentro de su alcance, el que hace compatible la obtención de la calidad, coste y plazo de ejecución, atendiendo a una determinada jerarquía que debe asignarse a cada uno de estos objetivos parciales.

3. TRADICIONALMENTE, EN OBRAS DE EDIFICACIÓN, EL SEGUIMIENTO Y CONTROL DE LA CONSTRUCCIÓN HA SIDO MISIÓN DE LA DIRECCIÓN FACULTATIVA. ¿SUPONE LA DIP LA DESAPARICIÓN DE ÉSTA?, ¿CÓMO SE ASUMEN LAS RESPONSABILIDADES LEGALES?.

Por supuesto, el Project Management oDirección Integrada de Proyecto, no supone la desaparición de las tradicionales direcciones facultativas; simplemente complementa el papel y la función de la Dirección Facultativa, ya que ésta tiene por misión únicamente el control técnico de la calidad, así como también complementar el proyecto en todo lo necesario para poder realizar la construcción y verificar las certificaciones de obra construida. Por otra parte, las responsabilidades legales que pertenecen al ámbito de la calidad de la construcción, no sólo de su ejecución sino también de la bondad del proyecto, 
permanecen íntegras con la(s) persona(s) que representa(n) o toma(n) la función de Dirección Facultativa.

A través de mi experiencia, he constatado que la forma óptima de dirigir de manera integrada o global una obra de construcción, es que las funciones de la Dirección Facultativa las asuma el Director de Proyecto ("Project Manager"); de esta manera no se rompe el sistema, cosa que ocurriría si se tratase de actuaciones separadas. Mi experiencia, referida a casos actuales, es que cuando las responsabilidades de la Dirección Facultativa y la de la Dirección Integrada de Proyecto están separadas, es que, a menos que exista una grandisima coordinación y deseos de colaboración mutua, se produce una disfunción debida a la rotura del sistema.

4. SUPONIENDO QUE SE TRATA DE UNA METODOLOGÍA DE GESTIÓN, ¿PUEDE DECIRNOS QUE TÉCNICAS SE UTILIZAN?.

La Dirección Integrada de Proyecto o Project Management es más que una metodología de gestión; es un sistema de dirección en el sentido del Management, que como ya se ha dicho, significa, en síntesis, optimizar todos los recursos de que se dispone. Las técnicas que utiliza la DIP son fundamentalmente las de realización de Estudios de Viabilidad(faceta importantisima, aunque frecuentemente olvidada) para la determinación de los objetivos del Proyecto; las correspondientes a Contratación y Compras, que son fundamentales para conocer cómo hay que solicitar ofertas y cómo debe responderse a las mismas; las de programación, en cualquiera de sus versiones, aunque todas ellas actualmente están basadas en las conocidas como CPM; también se utilizan técnicas de control de costes, pero en el bien entendido que de lo que se trata de hacer no es control presupuestario a posteriori, sino control de costes previsional para poder encajar la inversión dentro del objetivo programado,y, por tanto, lo que se hace y se utiliza tiene como finalidad realizar controles tanto del coste o del plazo de manera previsional, o sea que sirven de previsión para así poder efectuar las correcciones oportunas en cada momento. Otras técnicas utilizadas son las correspondientes a lafaceta técnica de control de calidad, con la consideración de que éstas deben de estar dentro del concepto de Calidad Total y por consiguiente de Garantía de Calidad. También se utilizan metodologías o técnicas de gestión del riesgo. Por último, también entran en la DIP para realizarla de manera eficaz, las técnicas de dirección de recursos humanos (motivación, liderazgo, creatividad,...) así como las de comunicación (informes de proyecto, reuniones,...). Igualmente se utilizan técnicas financieras, fundamentalmente en lo referente a la financiación del Proyecto. Como puede verse todas estas técnicas utilizadas conducen al cumplimiento de los objetivos mediante una optimización de recursos.

La DIP, por otra parte, utiliza el buen juicio y los

(c) Consejo Superior de Investigaciones Científicas conocimientos de toda índole para que la Propiedad o Patrocinador de un Proyecto, obtenga "lo mejor por lo que paga", o dicho de otra forma, la mejor relación calidad/ precio. En este sentido, también dentro de la DIP, se incluyen estudios de constructibilidad e ingeniería de valor que tienen por finalidad seleccionar los mejores procedimientos $\mathrm{y} / \mathrm{o}$ métodos constructivos, así como soluciones de proyecto referentes a disposición de elementos, materiales, etc... que optimicen los resultados.

5. SIENDO LA DIP ASÍ DE COMPLEJA, ¿CÓMO PUEDE ENTENDERSE QUE LA MAYORÍA DE LOS SUMINISTRADORES DE. "SOFTWARE" INFORMÁTICO ANUNCIAN QUE TIENEN PROGRAMAS CON LOS QUE SE REALIZA Y RESUELVE EL PROJECT MANAGEMENT DE CUALQUIER OBRA?

Es cierto que casi todas las sociedades que producen y/o comercializan "Software" Informático presentan programas de Project Management como una panacea para resolver todos los problemas que se presentan. Pero desgraciadamente no es cierto. Los programas informáticos al uso (unos mejores y más completos que otros) sólo inciden de manera general en la programación de tiempos y recursos. Hay programas del tipo "what if ", interactivos, que resultan muy útiles para la toma de decisiones cuando se producen desviaciones sobre los plazos previstos. Pero en todo caso, estos programas sólo constituyen una herramienta, imprescindible en Proyectos de gran complejidady/o magnitud, para hacer posible el tratamiento de muchos datos; aunque también debe advertirse que para proyectos simples y pequeños, la programación puede realizarse sin acudir a herramientas informáticas. En todo caso, repito, se trata de herramientas útiles que sólo son válidas para resolver una parte del complejo sistema que constituye la DIP.

6. SE PUEDE CONSIDERAR, QUE EN PRINCIPIO, LA APLICACIÓN DE ESTE SISTEMA DE GESTIÓN AUN PROYECTO CONCRETO REPRESENTA UN INCREMENTO DE LOS COSTES TRADICIONALES, ¿DE QUÉ FORMA SE PUEDEN CUANTIFICAR LAS VENTAJAS DEL PROJECT MANAGEMENT DENTRO DE LA INVERSIÓN TOTAL?; ¿SE TRATA SOLAMENTE DE ASPECTOS ECONÓMICOS?.

Con una mira corta, la aplicación de este sistema de dirección a un Proyecto concreto puede tomarse como que significa un incremento de costes, puesto que a todos los normales se añaden los honorarios correspondientes a la Dirección Integrada de Proyecto; ello no obstante, no significa una elevación del coste real, porque el simple hecho de poder cumplir con los objetivoso de quedarse muy cerca de ellos, económicamente tiene gran importancia. Si nos referimos al tiempo, cumplir el plazo en que se piensa ejecutar una obra tiene una importancia fundamental, ya 
que cualquier retraso significa también costes añadidos, producidos por el interés del dinero, que en la mayor parte de los casos dan lugar a un incremento de los mismos que superan con creces los honorarios de una Dirección Integrada de Proyecto. Por otra parte, cuando un Proyecto se realiza mediante DIP, se puede ejecutar bien mediante la metodología tradicional de contratar toda la obra con un contratista general, o bien la que permite la DIP, que es dividir el contrato en diversos "paquetes" o contratos parciales, en los que cada uno se contrata a su precio estricto; en este último caso, que es el que debe utilizarse cuando se emplea DIP como sistema directivo del Proyecto, se produce una fuerte economía debido a la desaparición de las cantidades que representan los "coeficientes de paso" o de "recargo" a que somete el contratista general el precio de cualquier subcontrato que realiza. En este sentido, podemos decir que se puede llegar al coste estricto de una obra, aunque en tal caso los honorarios de DIP puedan parecer elevados. No obstante, y según la experiencia que tenemos todos los que nos dedicamos a este tema, se pueden conseguir economías globales muy importantes.

Además, todo al final, se basa en aspectos económicos. El plazo significa dinero; el coste dinero en directo. Pero también, ejecutando una obra mediante un sistema de DIP, la Propiedad tiene en todo momento controlados todos los parámetros de la inversión; si la Dirección Integrada de Proyecto se aplica desde el origen, los tiene controlados incluso desde la etapa inicial del estudio de viabilidad y la del proyecto facultativo. Por añadidura, en esta fase del proyecto facultativo la DIP puede ejercer otras funciones muy importantes con el fin de mejorarlo; estas funciones son laIngeniería de Valor y los estudios de constructibilidad sobre el proyecto. La Ingeniería de Valor tiene como finalidad labúsqueday adopción de soluciones constructivas de igual funcionalidad y menor coste; los estudios de constructibilidad se utilizan para mejorar las soluciones constructivas. Mediante el uso de ambas no sólo se mejora el proyecto de ejecución, sino que también se consigue reducir el coste de la construcción.

7. EL HECHO DE UTILIZAR EL PROJECT MANAGEMENT ¿PUEDE COARTAR LA LIBERTAD DE DECISIÓN DE LAS PARTES IMPLICADAS EN UN PROYECTO PREVIAMENTE PLANIFICADO?. CONCRETAMENTE, ¿PUEDE IMPEDIR O DIFICULTAR DE ALGÚN MODO A LA PROPIEDAD REALIZAR CUALQUIER CAMBIO A LO LARGO DE LA VIDA DEL PROYECTO?.

Por supuesto, utilizar el Project Management no coarta ninguna libertad y pueden introducirse cambios a lo largo del ciclo de vida del Proyecto; lo que sucede, es que para que los cambios no incidan de manera importante 0 importantísima en un proyecto, tienen un período en el cual se pueden introducir con poco daño, otro en que el daño es mediano y otro, que es el final del proyecto, en que el daño producido por cualquier cambio es muy grande; concretando, si los cambios se introducen cuando sólo se está generando "papel" o sea durante la redacción del proyecto facultativo, tanto básico como de ejecución, los daños que un cambio puede inducir son pocos, incluso podría decirse que estos daños no son tal sino alteraciones, que en todo caso son controlables. Por el contrario, si los cambios aparecen cuando la obra ha comenzado su período de ejecución, la repercusión de los cambios comienza a tener importancia, que es tanto mayor cuanto más cerca se encuentra la obra de su finalización. Estos cambios repercuten en el coste de ejecución. Hay que tener en cuenta que todo lo que acabo de exponer sobre los cambios es en todo caso real, bien se apliquen sistemas de Dirección Facultativa tradicional, bien las actuales metodologías de DIP.

La ventaja frente a los cambios que ofrece la Dirección Integrada de Proyecto, es que como se está verificando un control continuo de todos los aspectos de la construcción, en todo momento se conoce el efecto de cualquier cambio y así se puede calibrar su importancia, no sólo la económica sino también la que produce sobre el plazo y sobre los demás aspectos del Proyecto; con ello se tienen conocimientos suficientes para decidir si el cambio es imprescindible o no. Además, disponer de un control continuo del Proyecto permite realizar dirección previsional, única manera de poder cumplir con los objetivos.

8. HISTÓRICAMENTE, ¿CUÁNDO, ENQUÉ PAÍSES Y EN QUÉ SECTORES COMENZÓ A APLICARSE ESTA TÉCNICA?. TENIENDO EN CUENTA QUE LA DIP SUPONE UN CONCEPTO INNOVADOR, ¿PUEDE CONSIDERARSE EN NUESTRO PAÍS QUE LA DIPES INNOVADORA?.

E1 Project Management, como disciplina absolutamente separada-disciplina que integra otras muchas - se empieza a aplicar hace unos 20 ó 25 años en los Estados Unidos de América. El sector en que comienza es el de la construcción de grandes proyectos muy complejos, tales como los de la NASA en sus ingenios espaciales; inmediatamente después comenzó a utilizarse en la construcción de plantas de proceso y centrales nucleares. Poco a poco, pero de manera bastante rápida, pasó a aplicarse en la construcción de edificios singulares, como hospitales, hoteles, escuelas y edificios de oficinas y análogos. En la construcción de viviendas todavía se utiliza poco. Desde los Estados Unidos de América se trasladó rápidamente al Reino Unido de la Gran Bretaña en donde, posiblemente por la gran tradición de utilizar consultores, se extendió con gran rapidez y tuvo una aceptación inmediata. En España comenzó a aplicarse hace unos 20 ó 25 años en el sectoror de construcción industrial y, concretamente, en el de lasplantas industriales, fundamentalmente las de proceso. Por consiguiente, 
podemos decir que esun sistema directivo de la construcción que se conoce perfectamente dentro del sector industrial; no obstante no ha trascendido todavía, de manera significativa, al sistema de construcción urbana y al de las obras públicas. Las razones habría que achacarlas quizáa que la construcción urbana, en general, obtiene grandes beneficios ajenos al proceso constructivo y las obras públicas se mueven dentro de una "cultura" que no es Empresarial sino la de la Administración del Estado, en donde a la optimización de recursos, hasta ahora, no se le ha dado ningunaimportancia.

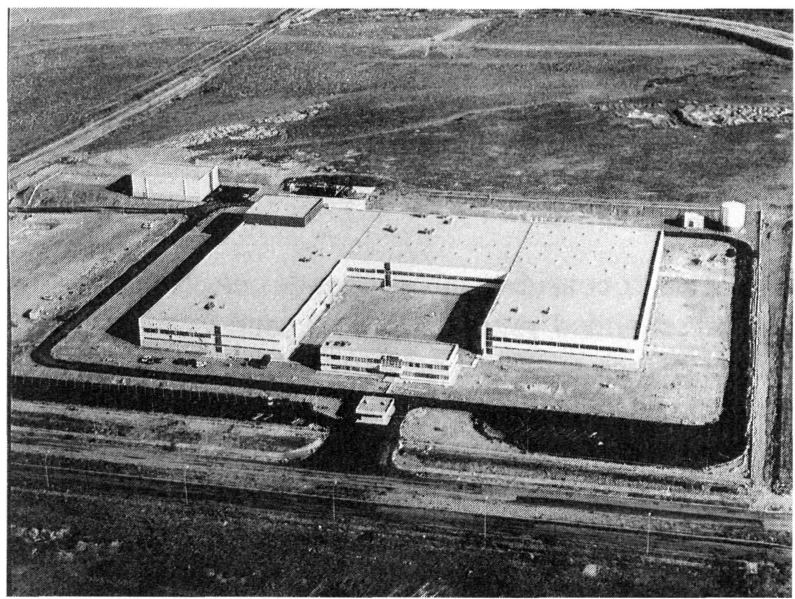

Planta de materiales "Composites". Toledo. Internacional de Composites. DFO y Construction Management: R. de Heredia.

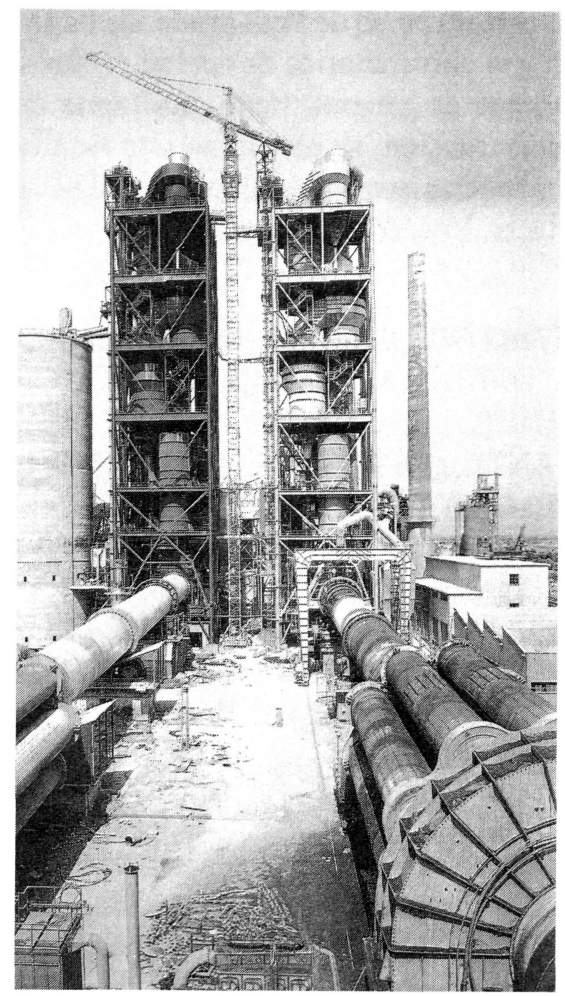

$2^{a}$ línea de clinker de la factoría El Alto, Valderribas, S. A. Proyecto: F.L. Smidth, S.A. DFO y Construction Management: R. de Heredia.
Por consiguiente, el Project Management o Dirección Integrada de Proyecto, en nuestro país se puede considerar un sistemainnovador sóloen los subsectores de laedificación urbana y de las obras públicas.

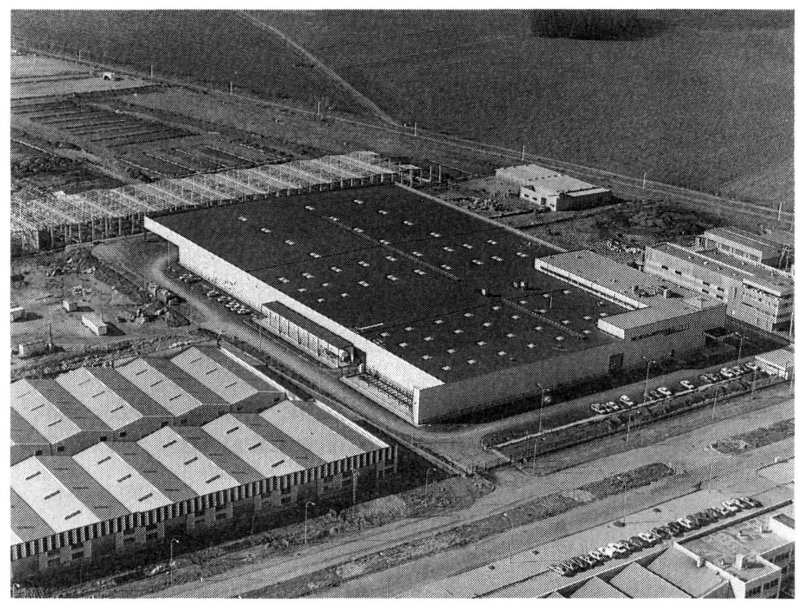

Planta de embotellado. La Casna (Toledo). Proyecto: R. de Heredia. DFO y Construction Management: R. de Heredia.

9. ¿A QUÉ OTRAS ACTIVIDADES O SECTORES DIFERENTES AL INDUSTRIAL Y AL DE LA CONSTRUCCIÓN CABRÍA APLICAR ESTA METODOLOGÍA?. ¿ES PATRIMONIO DE LA ACTIVIDAD PRODUCTIVA?. ¿HAY UN UMBRAL DE INVERSIÓN POR DEBAJO DEL CUAL NO TIENE SENTIDO APLICAR EL PROJECT MANAGEMENT?

¿A qué otras actividades se puede aplicar la DIP además de la construcción?. Si la DIP es dirigir Proyectos y Proyecto es cualquier actividad que está limitada en el tiempo y utiliza unos recursos diferenciados a través de una organización temporal, su campo de aplicación es casi ilimitado, ya que es aplicable a todo lo que sea un Proyecto.

¿Se aplicaen España? Yodiría que casi no. En construcción, que es donde más se utiliza como ya se ha dicho, su uso extensivo existe sólo en la industria de plantas de proceso y ahora comienza, tímidamente, autilizarse en la edificación industrial y urbana de edificios singulares; también se utiliza en proyectos de Defensa y en investigación en la industria farmacéutica. Evidentemente, en todo el mundo se está utilizando también en otros proyectos de $I+D$, de Informática, de Banca, de Automoción, de Publicidad, de Comunicaciones, ..., o sea en Proyectos de cualquier índole; incluso en las campañas políticas, ya que también son Proyectos.

Con respecto al umbral de inversión, puede determinarse conociendo el coste de la DIP y el beneficio económico que produce. Teniendo en cuenta únicamente este criterio, 


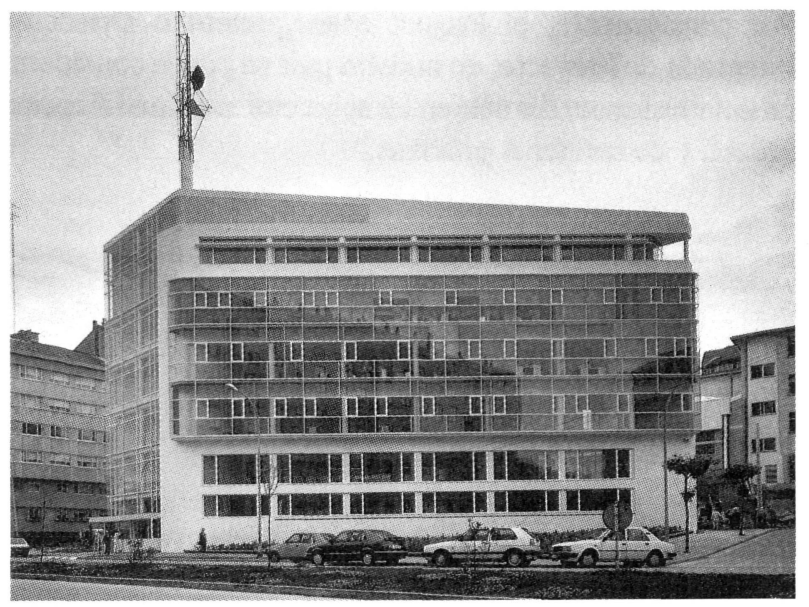

Centro Regional de Explotación del NO. de RedEléctrica de España (La Coruña). Proyecto: Andrés Perea, Arq. Construction Management: R. de Heredia.

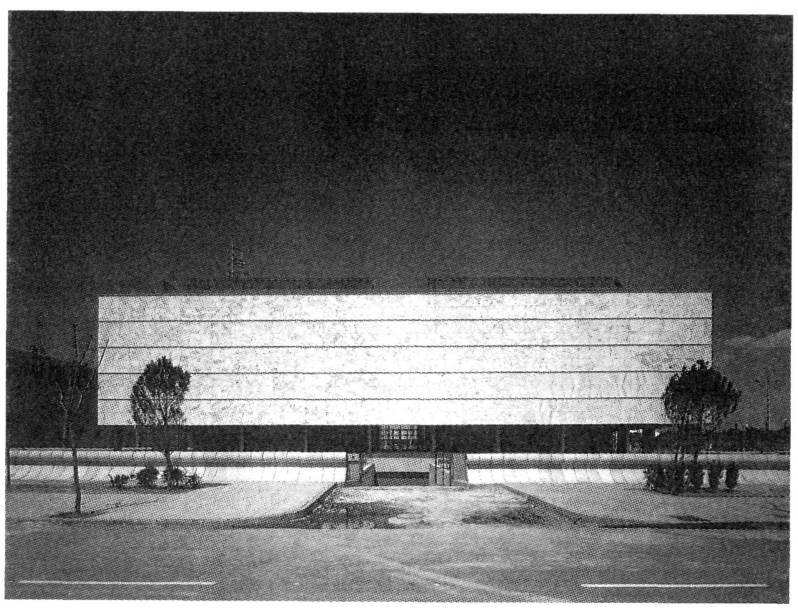

Centro Regional de Explotación de Red Eléctrica de España (Sevilla). Proyecto: Mariano Bayón, Arq. Construction Management: R. de Heredia.

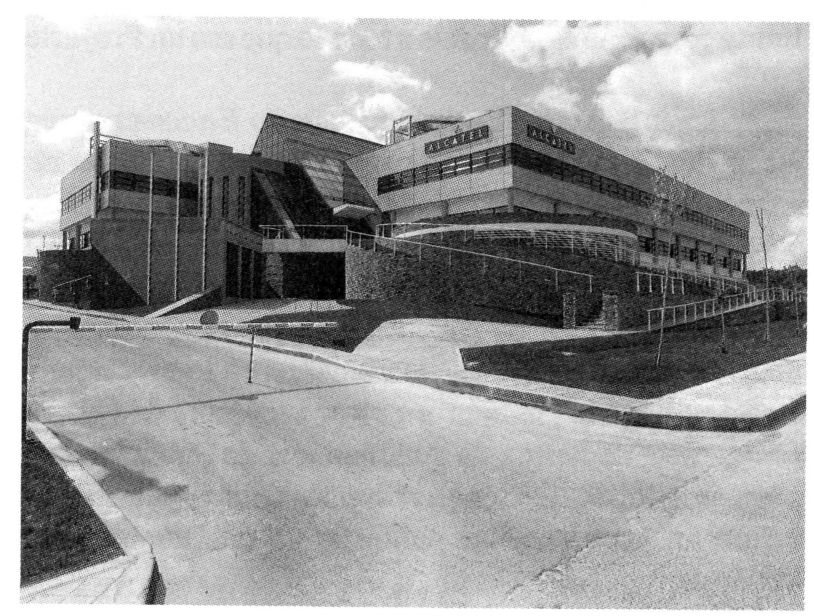

Centro de I+D de Alcatel. Tres Cantos (Madrid). Proyecto: F. Partearroyo, Arq. DFO y Construction Management: R. de Heredia. ahora, y para obras, podría decirse que cuandoel presupuesto es ya del orden de los 300 millones de pesetas, pueden resultar cifras muy alentadoras en cuanto a la economía que produce la DIP; así el umbral de inversión podría situarse en esta cifra. En inversiones menores, cuando la Propiedad/Promotor carece de experiencia y/o conocimientos sobre la DIP, estima que sus honorarios castigan la inversión, hecho que no es cierto ya que en todo caso la DIP produce ventajas comparativas frente a la forma tradicional de actuar que compensan sus costes.

10. ¿CONSIDERA QUE SE TRATA DE UNA DISCIPLINACON PERSONALIDADPROPIA AUNQUE SENUTRA DE OTRAS?; COMOTAL, ¿FORMA PARTE DE LAS ENSEÑANZAS DE LAS ESCUELAS TÉCNICAS?

Por supuesto, considero que se trata de una pluridisciplina con personalidad propia. En este sentido me gustaría indicar que se trata de un sistema directivo que utiliza metodologías y técnicas de Management y de Ingeniería.

Como enseñanza reglada dentro de las carreras universitarias de España, que yo conozca, sólo existe en la Escuela Técnica Superior de Ingenieros Industriales de la Universidad Politécnica de Madrid, donde se imparte dentro de mi Cátedra de Construcciones Industriales a los alumnos de $6^{\circ}$ año. Además existen programas de Postgrado que se imparten en Instituciones dedicadas, casi siempre, a formación de directivos. Dentro de mi Cátedra también existe un curso de Post-grado, de larga duración, dirigido hacia universitarios de cualquier titulación; este Curso, aunque es general, tiene una fuerte orientación hacia la construcción; su programa está inspirado en las últimas tendencias para la enseñanza de la DIP, generadas en los Estados Unidos de América.

11. DENTRO DEL COMPLEJO PROCESO QUE HOY DÍA ACOMPAÑA LA CONSTRUCCIÓN DE CUALQUIER OBRA MEDIANAMENTE IMPORT ANTE, ¿ES POSIBLE APLICAR EL PROJECT MANAGEMENT DE FORMA PARCIAL, O POR EL CONTRARIO, APLICARLO DE PRINCIPIO A FIN, O SEA ABARCANDODESDE LA CONCEPCIÓNHASTA EL INICIO DE LA EXPLOTACIÓN DEL PROYECTO? EL CONSTRUCTION MANAGEMENT, ¿ES UNA APLICACIÓN PARCIAL DEL PROJECT MANAGEMENT?

En una construcción, la DIP puede aplicarse y es lo ideal, en su sentido completo, o sea comenzando desde que se plantea una inversión; las primeras actividades de la DIP se realizan en la fase de Estudio de Viabilidad que es donde debe concebirse el Proyecto y generar sus objetivos. Ahora bien, con gran frecuencia, la DIP comienza a aplicarse sólo 
en algunas de las fases posteriores al inicio. La DIP completa se concibe vista desde el lado del Propietario/ Promotor; cuando éste es el caso, en construcción aparece el "Construction Management" - Dirección Integrada de Construcción - que solamente incluye algunas de las fases de la DIP; es por consiguiente un subsistema de ésta, en la que sus tareas fundamentales incluyen la dirección, el control, la ingeniería de valor y la constructibilidad del proyecto técnico, básico y de ejecución; el proceso de contratación de todas las obras; la preparación de las herramientas de control (de las que tiene una importancia sobresaliente la redacción de una correcta Estructura de Desagregación de Proyecto “EDP”); y el control y dirección previsional de la ejecución. Todo el proceso está dirigido por el Director de Proyecto (o Director de Construcción), que tiene como función intervenir en el Proyecto como elemento integrado en la Propiedad para aconsejar sobre las decisiones a tomar que mejoren la utilización de todos los recursos; a veces la Propiedad delega su autoridad en el Director Proyecto para que sea el responsable absoluto en la toma de decisiones.

Podemos así decir que el Construction Management es una aplicación parcial del Project Management, que es el que abarca el ciclo de vida completo de un Proyecto.

\section{2. ¿PUEDE EL PROJECT MANAGEMENT INTERFERIR EN LAS ACTIVIDADES TRADICIONALES DE TODOS LOS AGENTES QUE INTERVIENEN EN EL PROCESO DE CONSTRUCCIÓN?.}

Por supuesto que no. Puede interferir, pero en el sentido positivo, en el sentido de tener mayor coordinación, mayor control; de conseguir los objetivos del Proyecto, aún incluso cuando éstos no están explícitos, ya que una misión de la DIP/DIC es explicitarlos para lograrlos. En este sentido es importante resaltar que, en principio, excepto la Propiedad/ Promotor, los demás agentes que intervienen en el proceso constructivo, consideran en cualquier sistema directivo sólo los aspectos relacionados con el control, que a su vez lo ven como una amenaza. Realmente, como la finalidad de la DIP es realizar el Proyecto cumpliendo sus objetivos, nadie debiera ver la DIP como una amenaza, siempre y cuando todos tuvieran la intención de funcionar honestamente dentro de los términos de su contrato respectivo.

\section{Publicación del Instituto Eduardo Torroja-CSIC}

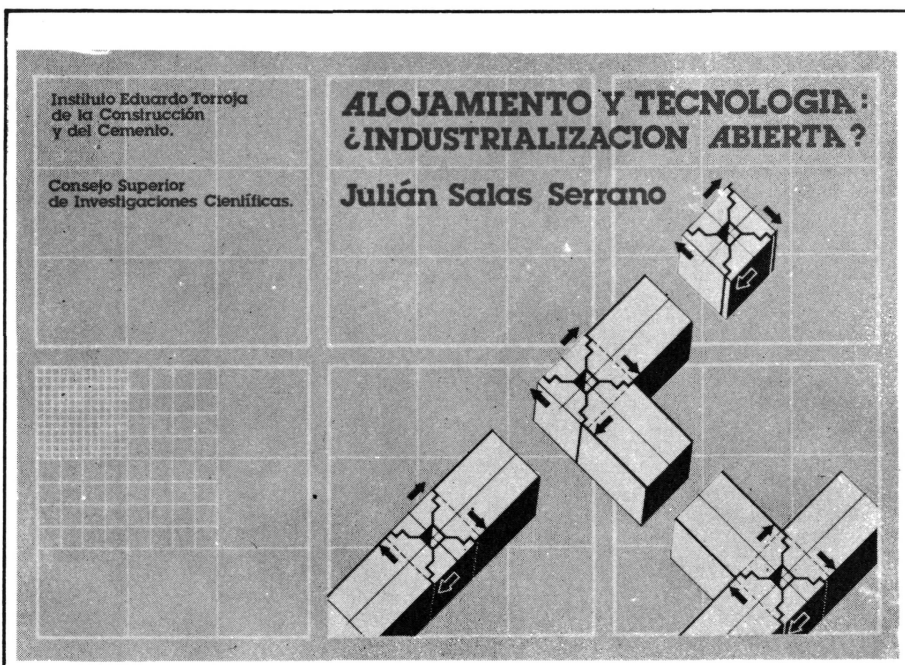

\section{ALOJAMIENTO Y TECNOLOGIA: ¿INDUSTRIALIZACION ABIERTA?}

JULIAN SALAS, ING. IND. (I.E.T.c.c.)

Un volumen de 160 páginas, 109 figuras y 16 tablas. Tamaño $240 \times 168 \mathrm{~mm}$. Encuadernado en rústica. Precios: España, 1.200 ptas; extranjero, 17 \$ USA.

\section{SUMARIO:}

Prólogo Prof. G. Ciribini.

\section{Introducción}

Capítulo 1.- La industrialización en las proclamas y manifiestos de arquitectura.

Capítulo 2. - ¿Réquiem por la construcción industrializada?

Capítulo 3. - Algunos conceptos básicos.

Capítulo 4.- ¿Proyecto tradicional, construcción industrializada?

Capítulo 5.-Componentes.

Capitulo 6. - La coordinación dimensional hoy

Capítulo 7.-Flexibilidad, intercambiabilidad y catálogos.

Capítulo 8. - Industrialización, normativa y calidad.

Capítulo 9.-Reflexiones finales.

publicación del

INSTITUTO EDUARDO TORROJA 